\title{
Overexpression of E3 ubiquitin ligase tripartite motif 32 correlates with a poor prognosis in patients with gastric cancer
}

\author{
MASAHIRO ITO, KAZUHIRO MIGITA, SOHEI MATSUMOTO, KOHEI WAKATSUKI, TETSUYA TANAKA, \\ TOMOHIRO KUNISHIGE, HIROSHI NAKADE, MITSUHIRO NAKATANI and YOSHIYUKI NAKAJIMA
}

Department of Surgery, Nara Medical University, Kashihara, Nara 634-8522, Japan

Received August 13, 2015; Accepted January 6, 2017

DOI: $10.3892 / 01.2017 .5806$

\begin{abstract}
Tripartite motif protein (TRIM) 32 belongs to the TRIM family, which is composed of RING finger, B-box and coiled-coil domains. TRIM32 has been reported to function as an enzyme 3 ubiquitin ligase and is overexpressed in numerous types of cancer. The present study evaluated the clinical significance of TRIM32 expression levels in gastric cancer. The current study also investigated the TRIM32 expression levels in 142 patients with gastric cancer using immunohistochemistry and examined its clinical importance and potential as a prognostic marker. Furthermore, the function of TRIM32 was examined in vitro. High TRIM32 expression levels were detected in gastric cancer tissues. The postoperative overall and relapse-free survival rates were significantly reduced in patients with tumors with high levels of TRIM32 expression compared with those with tumors expressing low levels of TRIM32. Tumors expressing high levels of TRIM32 were associated with an increased risk of postoperative recurrence, particularly hematogenous recurrence. Multivariate analysis identified TRIM32 status as an independent prognostic factor. Furthermore, TRIM32 gene silencing induced apoptosis and inhibited the proliferation of gastric cancer cells in vitro. Therefore, TRIM32 expression levels may be of potential prognostic value in gastric cancer.
\end{abstract}

\section{Introduction}

Gastric cancer is one of the most common types of cancer globally and is the second leading cause of cancer-associated mortality (1). Despite previous improvements in surgical techniques and the development of novel chemotherapeutic regimens, a number of patients present with recurrence following curative resection, particularly those with advanced

Correspondence to: Dr Masahiro Ito, Department of Surgery, Nara Medical University, 840 Shijo-cho, Kashihara, Nara 634-8522, Japan

E-mail:mito@naramed-u.ac.jp

Key words: enzyme 3 ubiquitin ligase, tripartite motif 32, gastric cancer, prognostic factor, hematogenous recurrence disease (2). Therefore, novel therapeutic strategies for gastric cancer are required. Furthermore, the identification of diagnostic markers to predict the outcomes of patients is important for establishing effective treatment strategies to improve prognosis.

The ubiquitin-proteasome system is a post-translational protein modification system that has been established as an underlying mechanism mediating protein degradation via the 26S proteasome (3). This system serves an important role in controlling protein turnover and regulating a number of signaling pathways and cellular processes $(4,5)$. The tripartite motif (TRIM) protein family is one of the E3 ubiquitin ligase complexes and was named due to its characteristic tripartite motif, which is composed of a RING finger domain, B1 or B2 boxes and a coiled-coil domain (6). TRIM family members have been established to be involved in numerous distinct biological processes, including cell growth, differentiation, development, muscular physiology and the innate immune response (6). Therefore, the dysfunction of TRIM family members has been associated with various diseases, including TRIM20 in familial Mediterranean fever, TRIM18 in X-linked Opitz/GBBB syndrome and TRIM37 in Mulibrey nanism (6). In addition, the up or downregulation of TRIM family members has been identified in several malignancies including TRIM19 in leukemia (7), TRIM24 in breast cancer (8) and TRIM25 in ovarian cancer (9).

TRIM32 is one of the TRIM family members and was first characterized in a yeast two-hybrid screen for proteins that bind to the trans-activator of transcription (Tat) protein, a protein that activates the transcription of lentiviruses (10). TRIM32 contains a characteristic tripartite motif that is shared with TRIM family members and has six repeats of the NHL (NCL-1, HT2A and LIN-41) motif (11). Human TRIM32 gene mutations have been detected in two genetic disorders, limb-girdle muscular dystrophy type 2H (11) and Bardet-Biedl syndrome (12). The overexpression of TRIM32 has been demonstrated in numerous types of human cancer, including lung and colorectal cancer and skin squamous cell, head and neck squamous cell and hepatocellular carcinoma (13-15). However, the expression levels of TRIM32 in gastric cancer remain to be established. Additionally, the clinical importance of TRIM32 expression levels in human cancer requires further elucidation. The focus of the present study was to investigate TRIM32 expression levels and its clinical relevance and 
prognostic potential in gastric cancer, by examining the role of TRIM32 in vitro using a small interfering RNA (siRNA) silencing technique.

\section{Materials and methods}

Patients and tissues. A total of 142 patients with pathological stage IB-IV gastric cancer (16) who underwent curative gastrectomy at the Department of Surgery, Nara Medical University (Nara, Japan) between January 2004 and December 2007 were enrolled in the current study. The selected patients did not receive chemotherapy or radiotherapy prior to the surgery. The patients included 105 males and 37 females with a mean age of 67.5 years (standard deviation, 11.4). The cancerous and non-cancerous tissues were obtained from resected patient specimens and were stored at $-80^{\circ} \mathrm{C}$. For the immunohistochemistry analysis, a section of each specimen was fixed in $10 \%$ phosphate-buffered formalin and embedded in paraffin. Tumors were classified according to the tumor-node-metastasis (TNM) staging system (16). The follow-up time was until March 2015 or the date of mortality. The mean follow-up time for all patients was 58.9 months (standard deviation, 30.7). Written informed consent was obtained from all patients prior to the operation and the study was conducted according to institutional ethical guidelines (Nara Medical University).

Immunohistochemistry analysis. Immunohistochemistry staining for TRIM32 was performed using a Dako EnVision ${ }^{\mathrm{TM}}$ system (Dako; Agilent Technologies, Inc., Santa Clara, CA, USA). The rabbit polyclonal anti-TRIM32 antibody (dilution, 1:200; cat. no. AB96612; Abcam, Tokyo, Japan) was used as the primary antibody. Formalin-fixed, paraffin-embedded samples of primary tumor were cut into $5 \mu \mathrm{m}$ sections, deparaffinized and rehydrated using a series of ethanol gradients. Antigen retrieval was performed by heating the tissue sections to $105^{\circ} \mathrm{C}$ for 20 min using a Target Retrieval Solution (pH 9.0; Dako; Agilent Technologies, Inc.). To block endogenous peroxidase activity, the sections were treated with a $0.3 \%$ solution of hydrogen peroxide in absolute methanol for $5 \mathrm{~min}$ at room temperature and were subsequently washed 3 times with PBS, for 5 min each time. Non-specific binding was blocked using PBS with 5\% goat serum (Dako; Agilent Technologies, Inc.) for $60 \mathrm{~min}$ at room temperature and followed by 3 PBS washes. The sections were incubated with the primary antibody overnight at $4^{\circ} \mathrm{C}$. Following 3 washes with PBS, the sections were detected using the EnVision ${ }^{\mathrm{TM}}+$ System horseradish peroxidase labeled polymer system (Dako; Agilent Technologies, Inc.) at $37^{\circ} \mathrm{C}$ for $30 \mathrm{~min}$ and washed 3 times with PBS. The reaction reagent used for antibody detection was 3,3'-diaminobenzidine tetrahydrochloride and the slides were counterstained with hematoxylin.

Review and scoring of immunohistochemistry stained sections. To evaluate the TRIM32 expression levels, images of cells were captured using a polarized light microscope (Carl Zeiss, Oberkochen, Germany), and $\geq 1,000$ tumor cells from the invasive front of tumors were scored at a magnification, $\mathrm{x} 400$ using AxioVision version 4.7.1 (Carl Zeiss). The expression levels of TRIM32 were semi-quantified using the histology $(\mathrm{H})$-score system as previously described (17). The intensity of the immunohistochemistry stain in the nuclei of cells was graded as follows: 0) No stain; i) weak stain; ii) strong stain. The percentage of stained cancer cells in each staining category was analyzed and the $\mathrm{H}$-score was calculated as follows: (Percentage of weak stained tumor cells) $+(2 x$ percentage of strong stained tumor cells). A threshold value for the $\mathrm{H}$-score for TRIM32 was selected to provide optimal separation between the low- and high-risk groups for overall survival rate.

Cell culture and transfection. The MKN45 (poorly differentiated adenocarcinoma) and MKN74 (moderately differentiated adenocarcinoma) human gastric cancer cell lines were obtained from the RIKEN BioResource Center (Tsukuba, Japan) and cultured in RPMI 1640 (Wako Pure Chemical Industries, Ltd., Osaka, Japan) supplemented with $10 \%$ fetal bovine serum (MP Biomedicals, Tokyo, Japan). All cells were maintained in an atmosphere containing $5 \% \mathrm{CO}_{2}$ at $37^{\circ} \mathrm{C}$. For transfection analysis, the MKN45 and MKN74 cells were transfected with control siRNA (Qiagen GmbH, Hilden, Germany) or $60 \mathrm{nM}$ of siRNA against TRIM32. The siRNA against TRIM32 was selected to target the following DNA sequence: 5'-GACCGTGGTAACTATCGTATA- 3 . When cells were $\sim 30 \%$ confluent, the siRNA transfections were performed using the Lipofectamine ${ }^{\circledR}$ system (Invitrogen; Thermo Fisher Scientific, Inc.) according to the manufacturer's protocol. A total of $72 \mathrm{~h}$ following transfection, the cells were then trypsinized and washed 3 times with PBS in preparation for quantitative polymerase chain reaction (qPCR) or western blot analysis and were also used for cell viability assays.

Extraction of total $m R N A$ and $q P C R$ analysis. Total RNA was isolated from MKN45 and MKN74 cells using the RNAspin Mini kit (GE Healthcare Life Sciences, Chalfont, UK), according to the manufacturer's protocol, and first-strand cDNA was synthesized from $1 \mathrm{mg}$ RNA using a High Capacity cDNA Reverse Transcription kit (Applied Biosystems; Thermo Fisher Scientific, Inc.) according to the manufacturer's protocol. For qPCR analysis, the cDNA was amplified using 2X TaqMan ${ }^{\circledR}$ Fast Universal PCR Master mix (Applied Biosystems; Thermo Fisher Scientific, Inc.) with TRIM32-specific (Hs00705875-s1; Applied Biosystems; Thermo Fisher Scientific, Inc.) and $\beta_{2}$-microgloblin-specific primers (Hs00984230-m1; Applied Biosystems; Thermo Fisher Scientific, Inc.) on the StepOnePlus ${ }^{\mathrm{TM}}$ Real-Time PCR system (Applied Biosystems; Thermo Fisher Scientific, Inc.) according to the manufacturer's protocol. The thermal cycling conditions were $95^{\circ} \mathrm{C}$ for $20 \mathrm{sec}$ followed by 40 cycles of $95^{\circ} \mathrm{C}$ for $1 \mathrm{sec}$ and $60^{\circ} \mathrm{C}$ for $20 \mathrm{sec}$. qPCR experiments for each gene were performed in triplicate. The expression levels of $\beta_{2}$-microglobulin, a housekeeping gene, were measured as a control with a standard curve to determine the integrity of the template RNA for all specimens. The ratio of the mRNA level of TRIM32 was calculated as follows: Absolute copy number of TRIM32/absolute copy number of $\beta_{2}$-microglobulin.

Western blot analysis. The proteins of the MKN45 and MKN74 cells were extracted using Pro-Prep ${ }^{\mathrm{TM}}$ Protein Extraction Solution (Intron Biotechnology, Inc., Seongnam, Korea). The protein concentration of the lysates was determined using a $\mathrm{BCA}^{\mathrm{TM}}$ protein assay kit (Thermo Fisher Scientific, Inc.). 
Equal amounts of proteins (MKN45 cells, $10 \mu \mathrm{g}$ for p53 and actin; $20 \mu \mathrm{g}$ for TRIM32, cleaved caspase 3 and cleaved PARP; MKN74 cells, $5 \mu \mathrm{g}$ for TRIM32, cleaved PARP and p53, $10 \mu \mathrm{g}$ for actin, and $20 \mu \mathrm{g}$ for cleaved caspase 3) were subjected to SDS-PAGE analysis using 10\% Mini Protean TGX ${ }^{\mathrm{TM}}$ precast polyacrylamide gel (Bio-Rad Laboratories, Inc., Hercules, CA, USA) and the proteins were transferred onto polyvinylidene difluoride membranes (Merck KGaA, Darmstadt, Germany). Actin was blotted for in all instances and all blots on the same membrane were of the same mass. Non-specific binding was blocked by incubation in $5 \%$ (w/v) skim milk powder in TBS-T for $1 \mathrm{~h}$ at room temperature and washed twice with TBS-T, for 10 min each time. A rabbit polyclonal anti-TRIM32 antibody (dilution, 1:500; cat. no. ab96612; Abcam), a mouse monoclonal anti-actin antibody (dilution, 1:100; cat. no. C-2; Santa Cruz Biotechnology, Inc., Dallas, TX, USA), a mouse monoclonal anti-p53 antibody (dilution, 1:100; cat. no. SC-126; Santa Cruz Biotechnology, Inc.), a rabbit monoclonal anti-cleaved poly (ADP-ribose) polymerase (PARP) antibody (dilution, 1:1,000; cat. no. 9541; Cell Signaling Technology, Inc., Danvers, MA, USA) and a rabbit monoclonal anti-cleaved caspase 3 antibody (dilution, 1:1,000; cat. no. 9664; Cell Signaling Technology, Inc.) were used as the primary antibodies. The membranes were incubated with the indicated primary antibodies overnight at $4^{\circ} \mathrm{C}$ and washed twice with TBS-T, for 10 min each time. Membranes were then incubated with goat anti-rabbit horseradish peroxidase (HRP)-conjugated Immunoglobulin $\mathrm{G}$ (IgG) (dilution, 1:5,000; cat. no. sc-2004; Santa Cruz Biotechnology, Inc.) or goat anti-mouse IgG-HRP (dilution, 1:5,000; cat. no. sc-2005; Santa Cruz Biotechnology, Inc.) for $1 \mathrm{~h}$ at room temperature and washed 3 times with TBS-T, for $10 \mathrm{~min}$ each time. The detection method used peroxidase activity on $\mathrm{X}$-ray films and was captured by an enhanced chemiluminescence detection system.

Cell viability assay. The cell viability was determined using the CellTiter-Blue ${ }^{\circledR}$ Cell Viability Assay kit (Promega Corporation, Madison, WI, USA), according to the manufacturer's protocol. Briefly, aliquots of $5 \times 10^{3} \mathrm{MKN} 45$ and MKN74 cells/well were cultured in 96 -well plates at $37^{\circ} \mathrm{C}$ for $24 \mathrm{~h}$ and subsequently the cells were transfected with control siRNA or TRIM32 siRNA as previously described. Following $72 \mathrm{~h}$ at $37^{\circ} \mathrm{C}$ of incubation, the CellTiter-Blue ${ }^{\circledR}$ reagent was added to each well and the cells were incubated at $37^{\circ} \mathrm{C}$ for an additional $2 \mathrm{~h}$. The fluorescence was measured at a wavelength of $590 \mathrm{~nm}$ upon excitation at $560 \mathrm{~nm}$ using a SoftMax ${ }^{\circledR}$ Pro 5 device (Molecular Devices, LLC, Sunnyvale, CA, USA). Each experiment was performed $\geq 3$ times.

Statistical analysis. Continuous variables were expressed as the mean \pm standard deviation and the means were compared using an unpaired $t$-test. Categorical variables were presented as numbers and percentages, and groups were compared using the $\chi^{2}$ test or Fisher's exact test. The overall survival rate was calculated from the length of time between the surgery and the date of mortality. The relapse-free survival rate was calculated from the length of time between the surgery and the relapse of the disease. The survival rate curves were expressed using the Kaplan-Meier estimate. The statistical difference between the survival rate curves was analyzed using the log-rank test. The univariate and multivariate hazard ratios (HR) were calculated using the Cox proportional hazards model. The statistical analyses were performed using SPSS version 19.0 (IBM SPSS, Armonk, NY, USA). Confidence intervals (CI) were calculated at $95 \%$. All significant variables in the univariate analysis were used in the multivariate analysis. $\mathrm{P}<0.05$ was considered to indicate a statistically significant difference.

\section{Results}

TRIM32 expression levels in human gastric cancer. The relative expression levels of TRIM32 in gastric cancer tissues were compared with non-cancerous gastric tissues. The results of qPCR analysis indicated that the gastric cancer tissues expressed increased levels of TRIM32 mRNA compared with the non-cancerous tissues ( $\mathrm{P}=0.001$; Fig. 1A). Notably, the TRIM32 expression levels in cancer tissues were increased compared with those of non-cancer tissues in 12/14 patients (85.7\%; Fig. 1B).

The TRIM32 protein expression levels in gastric cancer tissue specimens were analyzed using immunohistochemistry (Fig. 2). In all gastric cancer tissue specimens, positive staining for TRIM32 was present in the nuclei of cancer cells. Positive staining for TRIM32 was also detected in the cytoplasm of a number of cancer cells. In the normal gastric mucosa, positive staining for TRIM32 was not detected in the nuclei, but was present in the cytoplasm of a small number of cells. The mean percentage of weak and strong expressing TRIM32 tumor cells was $49 \%$ (standard deviation, 14.5) and $13.2 \%$ (standard deviation, 11.9), respectively (Fig. 2A-C). The mean H-score of TRIM32 expression was 75.4 (standard deviation, 25.5) in tumor cells.

To clarify the clinical significance and prognostic value of TRIM32 expression levels in gastric cancer, the specimens were classified into two groups according to the H-score of TRIM32 expression. The HR of mortality in the high TRIM32 expression group was highest when the threshold value of the $\mathrm{H}$-score was 84 (HR, 2.128) and, therefore, this was set as the threshold value for the $\mathrm{H}$-score. A total of 51 patients $(35.9 \%)$ with an $\mathrm{H}$-score of $\geq 84$ and 91 patients $(64.1 \%)$ with an $\mathrm{H}$-score $<84$ were classified into the TRIM32-low and TRIM32-high groups, respectively (Fig. 2D and E).

Clinicopathological significance of TRIM32 expression levels in human gastric cancer. The association between TRIM32 expression levels and clinicopathological characteristics is presented in Table I. There was no significant association between TRIM32 expression levels and a number of clinicopathological variables, including gender, age, tumor differentiation, tumor size, tumor depth, lymph node metastasis, distant metastasis, pathological stage and venous invasion.

Prognostic value of TRIM32 expression levels in gastric cancer. The prognostic value of TRIM32 expression levels in patients with gastric cancer was examined. The five-year overall survival rate was significantly lower in patients with TRIM32-high tumors compared with patients with TRIM32-low tumors (51.9\% vs. $75.5 \%$; $\mathrm{P}=0.008$; Fig. $3 \mathrm{~A})$. 

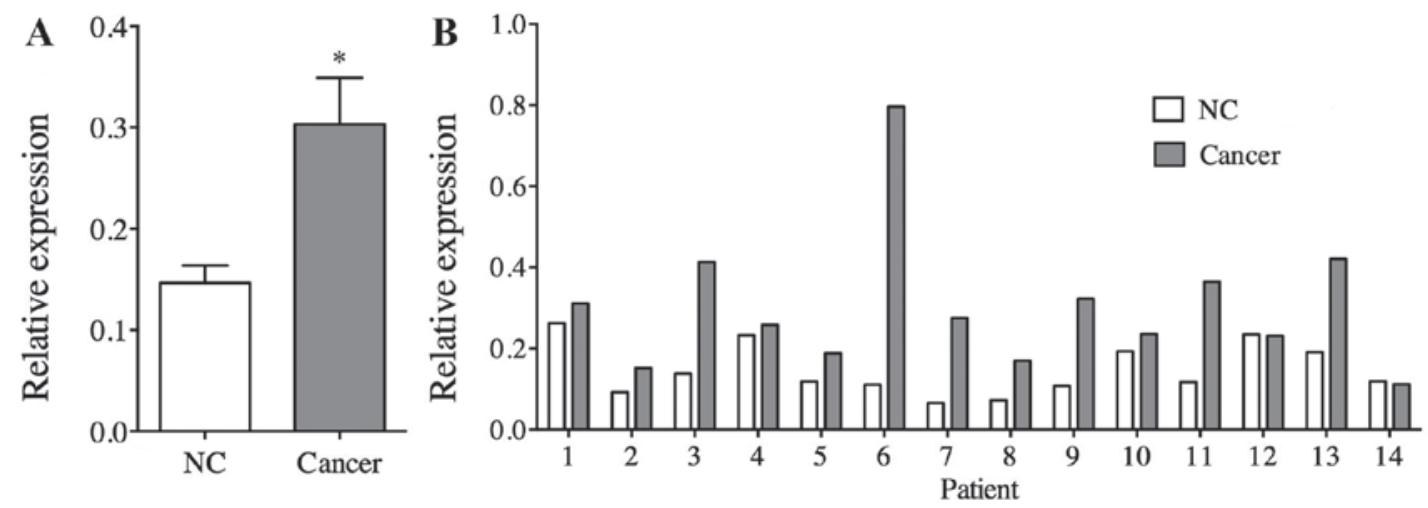

Figure 1. TRIM32 mRNA expression levels in gastric cancer tissues, as detected by reverse transcription-quantitative polymerase chain reaction. (A) Relative TRIM32 expression levels of cancer tissue specimens were significantly increased compared with that of non-cancerous tissues ( $\mathrm{n}=15$ for each group; $\mathrm{P}=0.011$ ). (B) In $86.7 \%$ of the patients with gastric cancer, the TRIM32 expression levels of cancer tissue specimens were increased compared with that of the NC tissue specimens. NC, non-cancerous; TRIM32, tripartite motif containing protein 32.

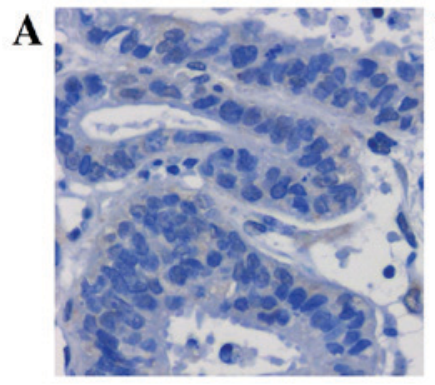

D

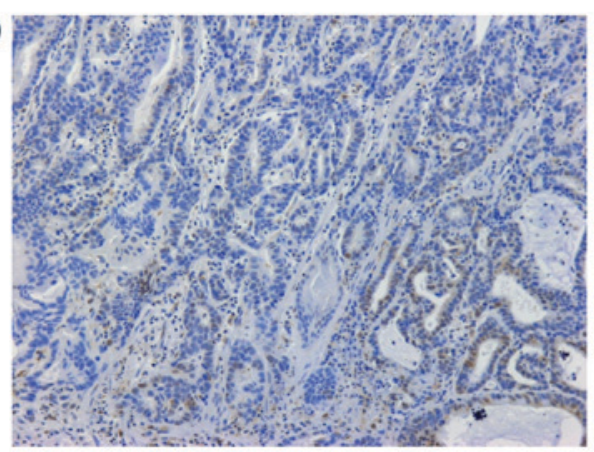

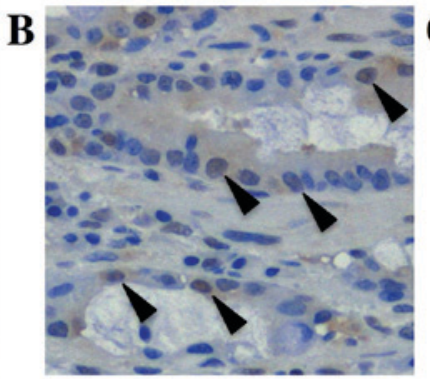
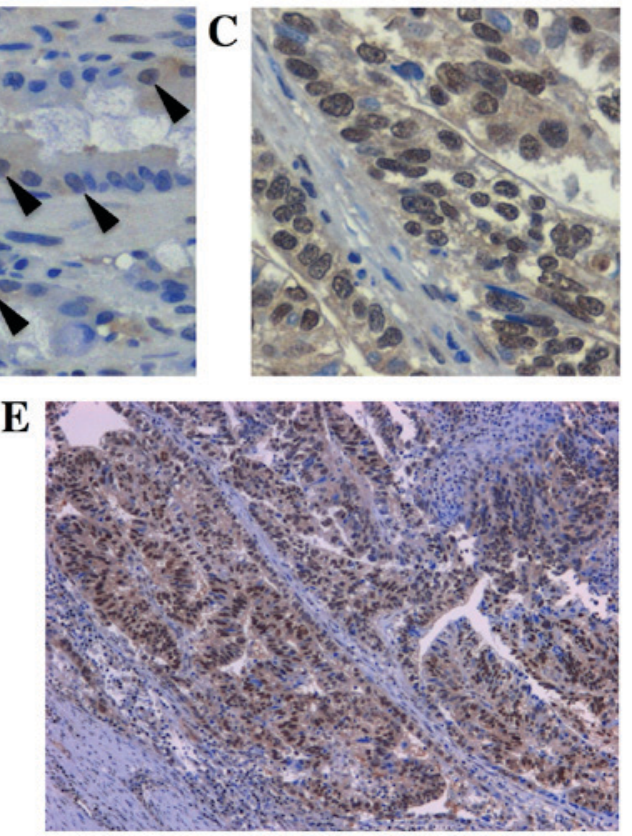

Figure 2. Differential expression of TRIM32 in gastric cancer tissue specimens. Immunohistochemistry staining of TRIM32 protein in tissue specimens with (A) non-reactive (0), (B) weak positive and (C) strong positive staining. Representative images of stain in cases of (D) low and (E) high expression levels of TRIM32. Black arrow heads are indicative of weak positive staining. Magnification, x100. TRIM32, tripartite motif containing protein 32.

According to univariate analysis, the HR for high TRIM32 expression was 2.128 (95\% CI, 1.197-3.783; P=0.01; Table II). The other characteristics that were significantly associated with the overall survival rate were the tumor size, tumor depth, lymph node metastasis, distant metastasis and venous invasion. The multivariate analysis demonstrated that the TRIM32 status was an independent prognostic factor for the overall survival rate (HR, 2.388; 95\% CI, 1.288-4.428; $\mathrm{P}=0.006$ ). These results suggest that TRIM32 may be a potential molecular prognostic marker for gastric cancer.

Influence of the TRIM32 expression levels on postoperative recurrence. The influence of the TRIM32 expression levels on postoperative recurrence was analyzed. As of March 2015, 38 patients $(26.8 \%)$ had developed postoperative tumor recurrence. The site of the primary relapse was to the peritoneum in
22 patients, hematogenous in 13 patients, to the lymph node in 5 patients and to the surrounding area in 1 patient. Overall, the rate of recurrence was increased in patients with TRIM32-high tumors compared with patients with TRIM32-low tumors ( $\mathrm{P}=0.034$; Table III). Notably, hematogenous recurrence was significantly higher in patients with TRIM32-high tumors compared with patients with TRIM32-low tumors $(\mathrm{P}=0.045)$. Furthermore, the five-year relapse-free survival rate was significantly reduced in patients with TRIM32-high tumors compared with patients with TRIM32-low tumors (51.2\% vs. 72.9\%; P=0.01; Fig. 3B).

\section{Downregulation of TRIM32 by siRNA inhibits cell proliferation} and induces apoptosis in human gastric cancer cells. In vitro experiments were performed to evaluate the role of TRIM32 in gastric cancer. MKN45 and MKN74 human gastric cancer 
Table I. Association between clinicopathological characteristics and TRIM32 expression levels.

\begin{tabular}{|c|c|c|c|c|}
\hline Variables & Number of patients & TRIM32-low ${ }^{\text {a }}(\%)$ & TRIM32-high ${ }^{\mathrm{b}} \%$ ) & P-value \\
\hline Gender & & & & $0.190^{c}$ \\
\hline Male & 105 & $64(70.3)$ & $41(80.4)$ & \\
\hline Female & 37 & $27(29.7)$ & $10(19.6)$ & \\
\hline Age, years ${ }^{\mathrm{d}}$ & & $68.4 \pm 10.7$ & $66.0 \pm 12.4$ & $0.223^{\mathrm{e}}$ \\
\hline Histology & & & & $0.704^{\mathrm{c}}$ \\
\hline Differentiated & 70 & $42(46.2)$ & $28(54.9)$ & \\
\hline Undifferentiated & 72 & $49(53.8)$ & $23(45.1)$ & \\
\hline Tumor size, $\mathrm{mm}^{\mathrm{d}}$ & & $47.1 \pm 33.1$ & $46.1 \pm 28.4$ & $0.862^{\mathrm{e}}$ \\
\hline Tumor depth & & & & $0.508^{\mathrm{f}}$ \\
\hline $\mathrm{T} 1$ & 29 & $12(13.2)$ & $7(13.7)$ & \\
\hline $\mathrm{T} 2$ & 49 & $33(36.3)$ & $16(31.4)$ & \\
\hline $\mathrm{T} 3$ & 41 & $24(26.4)$ & $17(33.3)$ & \\
\hline $\mathrm{T} 4$ & 33 & $22(24.1)$ & $11(21.6)$ & \\
\hline Lymph node metastasis & & & & $0.147^{\mathrm{c}}$ \\
\hline Negative & 50 & $36(39.6)$ & $14(27.5)$ & \\
\hline Positive & 92 & $55(60.4)$ & $37(72.5)$ & \\
\hline Distant metastasis & & & & $0.490^{\mathrm{f}}$ \\
\hline Negative & 135 & $87(95.6)$ & $48(94.1)$ & \\
\hline Positive & 7 & $4(4.4)$ & $3(5.9)$ & \\
\hline Pathological stage & & & & $0.101^{\mathrm{f}}$ \\
\hline IB & 42 & $29(31.9)$ & $13(25.5)$ & \\
\hline II & 51 & $35(38.5)$ & $16(31.4)$ & \\
\hline III & 42 & $23(25.3)$ & $19(37.2)$ & \\
\hline IV & 7 & $4(4.3)$ & $3(5.9)$ & \\
\hline Lymphatic invasion & & & & $0.013^{\mathrm{c}}$ \\
\hline Negative & 31 & $14(15.4)$ & $17(33.3)$ & \\
\hline Positive & 111 & $77(84.6)$ & $34(66.7)$ & \\
\hline Venous invasion & & & & $0.708^{\mathrm{a}}$ \\
\hline Negative & 89 & $56(61.5)$ & $33(64.7)$ & \\
\hline Positive & 53 & $35(38.5)$ & $18(35.3)$ & \\
\hline
\end{tabular}

${ }^{\mathrm{a}} \mathrm{n}=91 ;{ }^{\mathrm{b}} \mathrm{n}=51$ : ${ }^{\mathrm{c} I n d i c a t e s}$ data obtained by the $\chi^{2}$ test; ${ }^{\mathrm{d}}$ Indicates that data are expressed as the mean \pm standard deviation; ${ }^{\mathrm{e}}$ Indicates a value obtained by an unpaired $t$-test; f Indicates data obtained by Fisher's exact test. TRIM32, tripartite motif protein 32 .
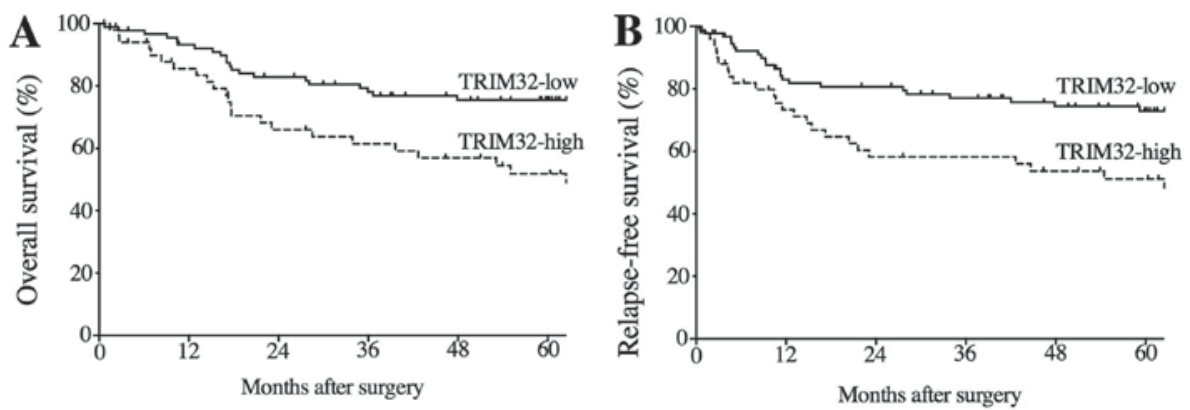

Figure 3. Postoperative survival rate was significantly reduced in patients with TRIM32-high tumors compared with those with TRIM32-low tumors. (A) Overall survival rate of patients with gastric cancer $(\mathrm{P}=0.008)$. (B) Relapse-free survival rate of patients with gastric cancer $(\mathrm{P}=0.01)$. TRIM32, tripartite motif containing protein 32 .

cell lines were examined to investigate the effects of TRIM32 downregulation via an siRNA knockdown. The TRIM32 expression levels were reduced at $72 \mathrm{~h}$ in the two cell lines following transfection of the TRIM32 siRNA (Fig. 4A and B). 
Table II. Results of univariate and multivariate analyses for patient survival rate.

\begin{tabular}{|c|c|c|c|c|}
\hline & \multicolumn{2}{|c|}{ Univariate analysis } & \multicolumn{2}{|c|}{ Multivariate analysis } \\
\hline & Hazard ratio $(95 \% \mathrm{CI})$ & P-value & Hazard ratio $(95 \% \mathrm{CI})$ & P-value \\
\hline Gender & 0.903 & & & \\
\hline Male vs. female & $(0.482-1.692)$ & 0.751 & & \\
\hline Age, years & 1.405 & & & \\
\hline$\geq 67$ vs. $<67$ & $(0.786-2.512)$ & 0.248 & & \\
\hline Tumor size, $\mathrm{mm}$ & 2.827 & & 2.298 & \\
\hline$\geq 50$ vs. $<50$ & $(1.587-5.035)$ & $<0.001$ & $(1.235-4.277)$ & 0.009 \\
\hline Tumor depth & 2.185 & & 1.160 & \\
\hline T3, T4 vs. T1, T2 & (1.194-3.997) & 0.009 & $(0.587-2.290)$ & 0.669 \\
\hline Lymph node metastasis & 3.603 & & 2.143 & \\
\hline Positive vs. negative & $(1.680-7.730)$ & $<0.001$ & $(0.961-4.776)$ & 0.062 \\
\hline Distant metastasis & 5.168 & & 2.978 & \\
\hline Positive vs. negative & $(2.013-13.267)$ & 0.005 & $(1.114-7.959)$ & 0.009 \\
\hline Lymphatic invasion & 1.390 & & & \\
\hline Positive vs. negative & $(0.669-2.890)$ & 0.377 & & \\
\hline Venous invasion & 2.353 & & 2.073 & \\
\hline Positive vs. negative & $(1.311-4.222)$ & 0.004 & $(1.075-3.998)$ & 0.030 \\
\hline TRIM32 expression & 2.128 & & 2.388 & \\
\hline High vs. low & $(1.197-3.783)$ & 0.010 & $(1.288-4.428)$ & 0.006 \\
\hline
\end{tabular}

Cox proportional hazards model was used to produce P-values. CI, confidence interval; TRIM32, tripartite motif protein 32.

Table III. Association of TRIM32 expression levels with postoperative tumor recurrence.

\begin{tabular}{lccccr}
\hline Site & TRIM32-low $^{\mathrm{a}}(\%)$ & TRIM32-high $^{\mathrm{b}}(\%)$ & Odds ratio & 95\% CI & P-value \\
\hline All recurrence & $19(20.9)$ & $19(37.3)$ & 2.250 & $1.052-4.812$ & $0.034^{\mathrm{c}}$ \\
Peritoneum & $11(12.1)$ & $11(21.6)$ & 2.000 & $0.799-5.008$ & $0.134^{\mathrm{c}}$ \\
Hematogenous & $5(5.5)$ & $8(15.7)$ & 3.200 & $1.025-10.370$ & $0.045^{\mathrm{d}}$ \\
Lymph node & $4(4.4)$ & $1(2.0)$ & 0.435 & $0.047-4.000$ & $0.408^{\mathrm{d}}$ \\
\hline
\end{tabular}

A number of patients had the first recurrence at $>1$ site. ${ }^{\mathrm{a}} \mathrm{n}=91 ;{ }^{\mathrm{b}} \mathrm{n}=51$; ${ }^{\mathrm{c}}$ Indicates data obtained by the $\chi^{2}$ test; ${ }^{\mathrm{d}}$ Indicates data obtained by Fisher's exact test. TRIM32, tripartite motif protein $32 ; \mathrm{CI}$, confidence interval.
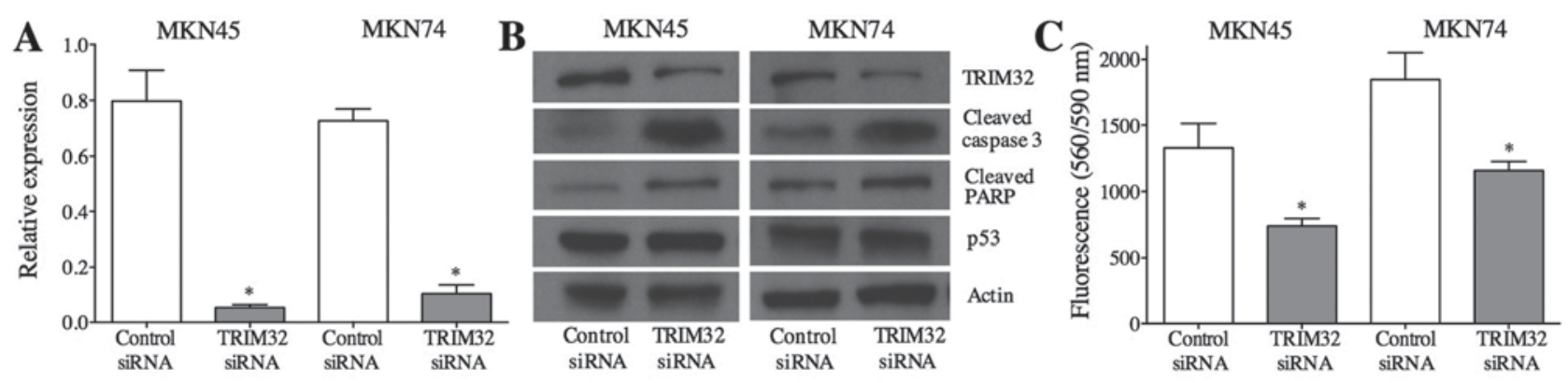

Figure 4. Downregulation of TRIM32 using siRNA inhibits the proliferation and increases the apoptosis of human gastric cancer cells. (A) MKN45 and MKN74 gastric cancer cells were transfected with control or TRIM32 siRNA. The expression levels of TRIM32 mRNA were reduced for $72 \mathrm{~h}$ in these cell lines following transfection of TRIM32 siRNA (n=3). (B) Expression levels of TRIM32, cleaved caspase 3, cleaved PARP, p53 and actin, as detected using western blot analysis in MKN45 and MKN74 cells following transfection with TRIM32 siRNA. (C) Cell proliferation was significantly reduced in cells treated with TRIM32 siRNA for $72 \mathrm{~h}$ compared with those treated with control siRNA, as determined using the CellTiter-Blue ${ }^{\circledR}$ Cell Viability assay (n=10). TRIM32, tripartite motif containing protein 32; siRNA, small interfering RNA; PARP, poly(ADP-ribose) polymerase; p53, protein 53. 
The role of TRIM32 in the regulation of cancer cell proliferation was examined by performing the CellTiter-Blue ${ }^{\circledR}$ Cell Viability assay. Cell proliferation was significantly suppressed following transfection with TRIM32 siRNA in MKN45 and MKN74 cells compared with cells transfected with the control RNA (Fig. 4C). Western blot analysis revealed that the protein expression levels of cleaved caspase 3 and cleaved PARP, which are apoptotic markers, were increased by TRIM32 siRNA in the two cell lines (Fig. 4B), whereas the levels of p53 remained unaltered. Therefore, these results suggest that TRIM32 serves an important role in regulating apoptosis and proliferation in gastric cancer cells.

\section{Discussion}

In the present study, the TRIM32 expression levels were investigated and its clinical relevance and prognostic value was evaluated in human gastric cancer. Previous studies have established that certain E3 ubiquitin ligases, including murine double minute 2 (18), S-phase kinase-associated protein 2 (19), Cullin1 (20) and Ring box protein 1 (RBX1) (21) were overexpressed in gastric cancer, and their overexpression was associated with poor patient prognosis. However, the expression levels of constitutive photomorphogenesis 1 (COP1) (22) and C-terminus of Hsc70-interacting protein (23) were reduced and this was associated with poor prognosis. Notably, the levels of RBX1 and COP1 expression were identified to be independent prognostic markers for gastric cancer $(21,22)$. A number of previous studies have identified aberrant expression levels of TRIM family members in gastric cancer $(24,25)$. TRIM28 (also termed Kruppel-associated box domain- associated protein 1) was identified to be overexpressed, and increased expression of TRIM28 was associated with a poor survival (24). However, TRIM29 expression levels have been revealed to be significantly decreased in gastric cancer tissues, compared with adjacent non-cancerous tissues and this decreased expression was associated with a poor survival (25). The role of TRIM32 in human gastric cancer remains to be elucidated. In the present study, TRIM32 was overexpressed in human gastric cancer tissue specimens and increased expression levels of TRIM32 were significantly associated with a poorer postoperative and overall survival rate. Furthermore, TRIM32 status was an independent predictor for the postoperative survival rate of patients with gastric cancer, independent of the TNM classification of the tumor. Therefore, the results indicate that TRIM32 may be a novel prognostic biomarker for gastric cancer.

The dysfunction of E3 ubiquitin ligases has been associated with recurrence in gastric cancer $(21,26)$. Huang et al (26) investigated autocrine motility factor receptor (AMFR) expression in 122 patients with gastric cancer and demonstrated that the overexpression of AMFR was significantly associated with a poor postoperative recurrence-free survival rate. Furthermore, this previous study suggested that the AMFR status was an independent predictor for gastric cancer recurrence following radical surgery (26). Another previous study identified that high RBX1 expression levels were associated with an increased risk of gastric cancer recurrence, particularly hematogenous recurrence (21). However, to the best of our knowledge, there have been no previous studies that have demonstrated an association between TRIM32 expression levels and postoperative recurrence in human cancer.

In the present study, increased TRIM32 expression levels were associated with a higher risk of gastric cancer recurrence. In particular, a significant association was identified between TRIM32 expression levels and postoperative hematogenous recurrence. These results suggest that TRIM32 may promote postoperative hematogenous recurrence of gastric cancer. However, the underlying mechanism by which TRIM32 affects hematogenous recurrence in gastric cancer remains to be elucidated, and further investigation is required.

E3 ubiquitin ligases have been established to regulate numerous oncogenes and tumor suppressors and their dysfunction has been associated with cancer cell proliferation (27). Previous studies have suggested that TRIM32 may be involved in cancer cell proliferation (14). Kano et al (14) demonstrated that TRIM32 increased the ubiquitination and degradation of Abelson interactor 2, which is a tumor suppressor that inhibits cell migration. This previous study also implicated that the overexpression of TRIM32 promoted cell growth, cell transformation and cell motility (14). In the present study, the association between TRIM32 expression levels and the proliferation of gastric cancer cells in vitro was investigated using siRNA knockdown, and the results identified that cell proliferation was significantly decreased upon suppression of TRIM32. Furthermore, the investigation of a potential mechanism underlying the inhibition of cell proliferation induced by TRIM32 silencing demonstrated that TRIM32 suppression induced apoptosis in gastric cancer cells, which was concordant with the increase in cleaved caspase 3 and cleaved PARP. Horn et al (13) investigated the association between TRIM32 expression levels and apoptosis signaling pathways and demonstrated that TRIM32 may be involved in cellular transformation and skin carcinogenesis via promoting the survival of cells over the induction of apoptosis (13). Liu et al (15) demonstrated that TRIM32 promotes p53 degradation via ubiquitination and that TRIM32 negatively regulates p53-mediated apoptosis, cell cycle arrest and senescence. Taken together, these results suggest that TRIM32 may negatively regulate cell proliferation and promote anti-apoptotic signaling pathways in gastric cancer. However, no significant differences in the expression levels of p53 protein were identified between cells treated with the TRIM32 siRNA and the control cells in the current study. Further investigation is required to elucidate the underlying mechanism by which TRIM32 is involved in cell proliferation and the apoptosis of gastric cancer cells.

In conclusion, to the best of our knowledge, the present study is the first to demonstrate that the overexpression of TRIM32 may be associated with an increased risk of postoperative recurrence and a poor prognosis in patients with gastric cancer. TRIM32 may serve an important role in regulating the proliferation of gastric cancer cells, possibly via its anti-apoptotic function. The results of the current study suggest that TRIM32 may be an effective prognostic biomarker for gastric cancer.

\section{Acknowledgements}

The authors of the current study would like to thank Japan Medical Communication (www.japan-mc.co.jp) for the English language editing. 


\section{References}

1. Parkin DM, Bray F, Ferlay J and Pisani P: Global cancer statistics, 2002. CA Cancer J Clin 55: 74-108, 2005.

2. Sun P, Xiang JB and Chen ZY: Meta-analysis of adjuvant chemotherapy after radical surgery for advanced gastric cancer. Br J Surg 96: 26-33, 2009.

3. Hershko A and Ciechanover A: The ubiquitin system. Annu Rev Biochem 67: 425-479, 1998.

4. Varshavsky A: The ubiquitin system, an immense realm. Annu Rev Biochem 81: 167-176, 2012.

5. Eldridge AG and O'Brien T: Therapeutic strategies within the ubiquitin proteasome system. Cell Death Differ 17: 4-13, 2010.

6. Reymond A, Meroni G, Fantozzi A, Merla G, Cairo S, Luzi L, Riganelli D, Zanaria E, Messali S, Cainarca S, et al: The tripartite motif family identifies cell compartments. EMBO J 20 2140-2151, 2001

7. Kakizuka A, Miller WH Jr, Umesono K, Warrell RP Jr, Frankel SR, Murty VV, Dmitrovsky E and Evans RM: Chromosomal translocation $\mathrm{t}(15 ; 17)$ in human acute promyelocytic leukemia fuses RAR alpha with a novel putative transcription factor, PML. Cell 66: 663-674, 1991.

8. Allton K, Jain AK, Herz HM, Tsai WW, Jung SY, Qin J, Bergmann A, Johnson RL and Barton MC: Trim24 targets endogenous p53 for degradation. Proc Natl Acad Sci USA 106: 11612-11616, 2009.

9. Sakuma M, Akahira J, Suzuki T, Inoue S, Ito K, Moriya T, Sasano H, Okamura K and Yaegashi N: Expression of estrogen-responsive finger protein (Efp) is associated with advanced disease in human epithelial ovarian cancer. Gynecol Oncol 99: 664-670, 2005.

10. Fridell RA, Harding LS, Bogerd HP and Cullen BR: Identification of a novel human zinc finger protein that specifically interacts with the activation domain of lentiviral Tat proteins Virology 209: 347-357, 1995.

11. Albor A, El-Hizawi S, Horn EJ, Laederich M, Frosk P, Wrogemann K and Kulesz-Martin M: The interaction of Piasy with Trim32, an E3-ubiquitin ligase mutated in limb-girdle muscular dystrophy type $2 \mathrm{H}$, promotes Piasy degradation and regulates UVB-induced keratinocyte apoptosis through NFkappaB. J Biol Chem 281: 25850-25866, 2006.

12. Watanabe T, Imoto I, Kosugi Y, Ishiwata I, Inoue S, Takayama M, Sato A and Inazawa J: A novel amplification at 17q21-23 in ovarian cancer cell lines detected by comparative genomic hybridization. Gynecol Oncol 81: 172-177, 2001.

13. Horn EJ, Albor A, Liu Y, El-Hizawi S, Vanderbeek GE, Babcock M, Bowden GT, Hennings H, Lozano G, Weinberg WC and Kulesz-Martin M: RING protein Trim32 associated with skin carcinogenesis has anti-apoptotic and E3-ubiquitin ligase properties. Carcinogenesis 25: 157-167, 2004.

14. Kano S, Miyajima N, Fukuda S and Hatakeyama S: Tripartite motif protein 32 facilitates cell growth and migration via degradation of Abl-interactor 2. Cancer Res 68: 5572-5580, 2008.
15. Liu J, Zhang C, Wang XL, Ly P, Belyi V, Xu-Monette ZY, Young KH, Hu W and Feng Z: E3 ubiquitin ligase TRIM32 negatively regulates tumor suppressor p53 to promote tumorigenesis. Cell Death Differ 21: 1792-1804, 2014

16. Sobin L, Gospodarowicz M and Wittekind C: TNM classification of malignant tumours. 7th edition. New Jersey, Wiley-Blackwell, 2009.

17. Robertson JF, Nicholson RI, Bundred NJ, Anderson E, Rayter Z, Dowsett M, Fox JN, Gee JM, Webster A, Wakeling AE, et al: Comparison of the short-term biological effects of 7alpha[9-(4,4,5,5,5-pentafluoropentylsulfinyl)-nonyl]estra-1,3,5, (10)-triene-3,17beta-diol (Faslodex) versus tamoxifen in postmenopausal women with primary breast cancer. Cancer Res 61: 6739-6746, 2001

18. Ye Y, Li X, Yang J, Miao S, Wang S, Chen Y, Xia X, Wu X, Zhang J, Zhou Y, et al: MDM2 is a useful prognostic biomarker for resectable gastric cancer. Cancer Sci 104: 590-598, 2013.

19. Masuda TA, Inoue $H$, Sonoda H, Mine S, Yoshikawa $Y$, Nakayama K, Nakayama K and Mori M: Clinical and biological significance of S-phase kinase-associated protein 2 (Skp2) gene expression in gastric carcinoma: Modulation of malignant phenotype by Skp2 overexpression, possibly via p27 proteolysis. Cancer Res 62: 3819-3825, 2002.

20. Bai J, Zhou Y, Chen G, Zeng J, Ding J, Tan Y, Zhou J and Li G: Overexpression of Cullin 1 is associated with poor prognosis of patients with gastric cancer. Hum Pathol 42: 375-383, 2011.

21. Migita K, Takayama T, Matsumoto S, Wakatsuki K, Tanaka T, Ito M, Nishiwada S and Nakajima Y: Prognostic impact of RING box protein-1 (RBX1) expression in gastric cancer. Gastric Cancer 17: 601-609, 2014.

22. Sawada G, Ueo H, Matsumura T, Uchi R, Ishibashi M, Mima K, Kurashige J, Takahashi Y, Akiyoshi S, Sudo T, et al: Loss of COP1 expression determines poor prognosisin patients with gastric cancer. Oncol Rep 30: 1971-1975, 2013.

23. Gan L, Liu DB, Lu HF, Long GX, Mei Q, Hu GY, Qiu H and Hu GQ: Decreased expression of the carboxyl terminus of heat shock cognate 70 interacting protein in human gastric cancer and its clinical significance. Oncol Rep 28: 1392-1398, 2012

24. Yokoe T, Toiyama Y, Okugawa Y, Tanaka K, Ohi M, Inoue Y, Mohri Y, Miki C and Kusunoki M: KAP1 is associated with peritoneal carcinomatosis in gastric cancer. Ann Surg Oncol 17: 821-828, 2010.

25. Kosaka Y, Inoue H, Ohmachi T, Yokoe T, Matsumoto T, Mimori K, Tanaka F, Watanabe M and Mori M: Tripartite motif-containing 29 (TRIM29) is a novel marker for lymph node metastasis in gastric cancer. Ann Surg Oncol 14: 2543-2549, 2007.

26. Huang Z, Zhang N, Zha L, Mao HC, Chen X, Xiang JF, Zhang H and Wang ZW: Aberrant expression of the autocrine motility factor receptor correlates with poor prognosis and promotes metastasis in gastric carcinoma. Asian Pac J Cancer Prev 15: 989-997, 2014.

27. Schlabach MR, Luo J, Solimini NL, Hu G, Xu Q, Li MZ, Zhao Z, Smogorzewska A, Sowa ME, Ang XL, et al: Cancer proliferation gene discovery through functional genomics. Science 319: 620-624, 2008. 